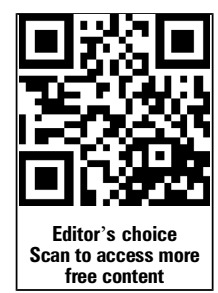

free access more free content

\title{
Unruptured intracranial aneurysms conservatively followed with serial CT angiography: could morphology and growth predict rupture?
}

\author{
William A Mehan Jr, ${ }^{1}$ Javier M Romero, ${ }^{1}$ Joshua A Hirsch, ${ }^{1}$ David J Sabbag, ${ }^{2}$ \\ Ramon G Gonzalez, Jeremy J Heit, ${ }^{1}$ Pamela W Schaefer ${ }^{1}$
}

${ }^{1}$ Department of Radiology, Massachusetts General Hospital, Harvard Medical School, Boston, Massachusetts, USA

${ }^{2}$ Department of Radiology, Baylor College of Medicine, Houston, Texas, USA

\section{Correspondence to} Dr William A Mehan Jr, Department of Radiology, Division of Neuroradiology, Massachusetts General Hospital, Gray B285, Fruit Street, Boston, MA 02114, USA will.mehan@gmail.com

Received 25 August 2013 Revised 2 November 2013 Accepted 5 November 2013 Published Online First 25 November 2013

\section{CrossMark}

To cite: Mehan Jr WA, Romero JM, Hirsch JA, et al. J Neurolntervent Surg 2014:6:761-766.

\section{ABSTRACT}

Background and purpose Despite several landmark studies, the natural history of unruptured intracranial aneurysms (UIA) remains uncertain. Our aim was to identify or confirm factors predictive of rupture of UIA being observed conservatively with serial $\mathrm{CT}$ angiography (CTA) in a North American patient population.

Methods We performed a retrospective review of patients with UIA being followed with serial CTA studies from 1999 to 2010. The following features for each aneurysm were cataloged from the official radiologic reports and CTA images: maximum diameter, growth between follow-up studies, location, multiplicity, wall calcification, intraluminal thrombus and morphology. Univariate logistic regression analysis of the potential independent risk factors for aneurysm rupture was performed. Statistically significant risk factors from the univariate analysis were then entered into a multivariate logistic regression analysis.

Results 152 patients with a total of 180 UIA had at least two CTA studies. Six aneurysms in six different patients ruptured during the CTA follow-up period for an overall rupture rate of $3.3 \%$ and an annual rupture rate of $0.97 \%$. All ruptured aneurysms were $\geq 9 \mathrm{~mm}$. In the univariate analysis, the statistically significant predictors of aneurysm rupture were aneurysm size $(p=0.003)$, aneurysm growth $(p<0.0001)$ and aneurysm multilobulation ( $p=0.001)$. The risk factors that remained significant following the multivariate analysis were growth (OR 55.9; 95\% Cl 4.47 to 700.08 ; $\mathrm{p}=0.002$ ) and multilobulation (OR $17.4 ; 95 \% \mathrm{Cl} 1.52$ to 198.4; $p=0.022$ ).

Conclusions Aneurysm morphology and interval growth are characteristics predictive of a higher risk of subsequent rupture during conservative CTA follow-up.

\section{INTRODUCTION}

The natural history of unruptured intracranial aneurysms (UIA) is uncertain. The relative lack of clearly defined features that predict rupture renders the decision-making process of whether to surgically treat or conservatively manage an aneurysm complicated. The decision to treat or follow an UIA must be based on whether the treatment benefit outweighs the risk of rupture at a later date. Knowledge of aneurysm characteristics predictive of a higher risk of rupture may help to clarify this decision-making process.

UIA are relatively common. They occur in approximately $3-6 \%$ of the population based on imaging and autopsy studies. ${ }^{1-3}$ As minimally invasive techniques such as MR angiography (MRA) and CT angiography (CTA) have improved and are increasingly used for the evaluation of a variety of neurologic conditions, larger numbers of UIA are being identified. ${ }^{4}$ Risk stratification for UIA is important because aneurysm rupture is associated with significant morbidity and mortality. Aneurysms smaller than $7 \mathrm{~mm}$ have been shown to have a lower risk of rupture with a higher risk associated with posterior circulation (including posterior communicating artery) aneurysms. ${ }^{5}$ Modifiable patient risk factors associated with the rupture of intracranial aneurysms include cigarette smoking and hypertension. ${ }^{5-8}$

To our knowledge, only two studies have investigated the use of CTA exclusively to follow up UIA to better predict the risk factors for aneurysm rupture. One study was performed in a population of Japanese patients and found that a history of subarachnoid hemorrhage, large size (>10 mm) and posterior circulation location were significant independent predictors of aneurysm rupture. ${ }^{9}$ It has been demonstrated that people of Japanese descent have a genetic predisposition to intracranial aneurysm formation and a higher rate of aneurysm rupture, making this population inherently different from North American patients. ${ }^{10} 11$ An additional recent study examined the CTA features of asymptomatic unruptured UIA that predicted aneurysm growth specifically as it relates to subsequent rupture; aneurysm growth was found to confer a 12 -fold risk of rupture. ${ }^{12}$ However, this study did not analyze other aneurysm factors on CTA that may be predictive of rupture during conservative observation.

We acknowledge that previous multicenter prospective studies, such as those performed by the International Study of UIA (ISUIA), the Unruptured Cerebral Aneurysm Study of Japan (UCAS) and the Small UIA Verification Study (SUAVe) of Japan, demonstrated characteristics of UIA that are associated with rupture. $^{5} 1314$ While imperfect, their prospective design provides helpful information by de facto limiting selection bias. However, these trials used a heterogeneous mixture of imaging modalities including catheter digital subtraction angiography (DSA) to follow up aneurysms.

The purpose of our study is to identify specific imaging features that indicate an increased risk for the rupture of UIA being followed conservatively 
with serial CTA in a North American patient population at a single hospital center.

\section{METHODS}

\section{Patient selection}

We performed a retrospective analysis of patients with UIA. Using a proprietary data-mining tool, we searched through our Institution's radiology database to identify all patients who had undergone multiple CTA examinations between January 1999 and December 2010. Inclusion criteria were adult patients (age $\geq 19$ years) with at least one UIA who were followed with at least two serial CTA examinations performed over a period of at least 12 months. Exclusion criteria were: (1) previous history of aneurysmal subarachnoid hemorrhage; (2) history of treatment of an intracranial aneurysm (endovascular or open surgical) prior to the study; (3) presence of an additional vascular malformation (eg, arteriovenous malformation); (4) presence of mycotic, tiny $(<2 \mathrm{~mm})$ and/or fusiform aneurysms; and (5) follow-up examination within 12 months of the first examination. The electronic longitudinal medical record was reviewed for every patient included in our study to document demographics, smoking status and hypertension during the study period.

At our Institution the decision to recommend treatment or conservative imaging follow-up for UIA was determined by a multidisciplinary conference comprised of neurosurgeons, neurointerventional radiologists and neurologists. Decisions to treat aneurysms were based on consideration of aneurysm size, growth, complexity of treatment, patient age and overall health.

\section{Image acquisition}

CTA studies were performed at approximately 12 -month intervals for the evaluation of UIA being observed conservatively. For patients followed over many years the frequency of CTA imaging was decreased over time if an aneurysm was stable.

Multidetector CTA on either 16- or 64-detector row units was performed by scanning from the base of the $\mathrm{C} 1$ vertebral body to the vertex using an axial technique with 0.5 pitch, $1.25 \mathrm{~mm}$ collimation, 350 maximal mA, $120 \mathrm{kVp}, 22 \mathrm{~cm}$ field of view and $65-85 \mathrm{~mL}$ of iodinated contrast material administered by power injector at $4-5 \mathrm{~mL} / \mathrm{s}$ into an antecubital vein with either a fixed $25 \mathrm{~s}$ delay between the onset of contrast injection and the start of scanning or Smart-Prep, a semiautomatic contrast bolus triggering technique (General Electric Medical Systems, Waukesha, Wisconsin, USA). The resulting $1.25 \mathrm{~mm}$ thick axial source images were digitally archived. Maximum intensity projection (MIP) images of the major intracranial vessels were created in axial, coronal and sagittal planes.

\section{Study interpretation}

For each patient and each intracranial aneurysm the official CTA radiologic report was reviewed by a neuroradiologist (WAM, fellow) and the following aneurysm features were recorded: (1) maximum aneurysm diameter (in any plane); (2) aneurysm location: anterior cerebral artery (ACA), middle cerebral artery (MCA), internal carotid artery (ICA) and vertebrobasilar artery; and (3) single versus multiple aneurysms. Subsequently, three neuroradiologists (WAM), JMR (attending with 10 years of experience) and JAH (fellow)) independently reviewed the CTA images (axial source, MIP and three-dimensional images) for each case on picture archiving and communications system. The reviewers, blinded to the official radiologic report at the time of image analysis, cataloged the presence or absence of the following features: (1) interval growth of the aneurysm between follow-up CTA studies; (2) calcification within the aneurysm wall; (3) aneurysm intraluminal thrombus; and (4) aneurysm sac configuration (unilobular vs multilobular). The presence or absence of aneurysm rupture during the CTA follow-up period was confirmed by reviewing the medical records.

The period of observation for each aneurysm was defined as the time between the initial CTA and the last follow-up CTA for each patient in the interval from January 1999 to December 2010. If an aneurysm was treated during the study period, only the CTA studies prior to treatment were included in the analysis. For aneurysm location, the anterior circulation was considered to be anywhere within the ACA, MCA or ICA distribution and the posterior circulation was considered to be anywhere within the vertebrobasilar system, posterior cerebral artery branches and the posterior communicating arteries. For aneurysm growth, an increase in measured size of the aneurysm of $>2 \mathrm{~mm}$ in any dimension (anterior-posterior, transverse or superior-inferior) between studies was considered to be enlargement. If an aneurysm grew during follow-up, the maximum diameter during the study period was considered to be the size of the aneurysm. The morphologic parameter multilobulated was defined as the presence of daughter sacs, lobules or lobulation of the aneurysm sac. Aneurysm calcification and thrombus were defined as the presence of any mural calcification or luminal thrombus within the aneurysm sac, respectively.

\section{Statistical analysis}

Data were analyzed with the XLSTAT statistics program (Addinsoft, USA). Patient and aneurysm categorical and continuous variables that may be predictive of aneurysm rupture during follow-up were analyzed using a logistic regression model. Univariate analyses were first performed for each independent variable. All univariate parameters found to be statistically significant were then used in a multivariate logistic regression model. Independent variables were analyzed using the Wald test. ORs and 95\% CIs for each independent factor associated with aneurysm rupture were calculated. A $p$ value of $<0.05$ was considered significant.

\section{RESULTS}

During the study period a total of 3033 cerebral aneurysms were referred to our institution, of which 614 ruptured. A total of 152 patients with 180 UIA met our inclusion criteria (table 1); 29 patients with 35 UIA were excluded from our analysis since they were followed for less than 12 months by CTA. By chance, none of the patients in our population was Japanese.

A total of six aneurysms in six different patients ruptured during the CTA follow-up period, yielding an overall rupture rate of $3.3 \%$ and an annual rupture rate of $0.97 \%$ (table 2). We initially performed a univariate logistic regression analysis of potential risk factors for aneurysm rupture (table 1). All six ruptured aneurysms were at least $9 \mathrm{~mm}$ in maximum diameter (mean $13.7 \mathrm{~mm}$; range 9-22 $\mathrm{mm}$ ) compared with a mean of $5.4 \mathrm{~mm}$ (range $2-35 \mathrm{~mm}$ ) for the unruptured aneurysms $(p=0.003)$. Four of six $(66.7 \%)$ ruptured aneurysms demonstrated interval growth by the time of rupture compared with 4 of $174(2.3 \%)$ unruptured aneurysms $(p<0.0001$, figure 1$)$. In one of the four ruptured aneurysms that enlarged between serial CTA follow-up studies, interval growth was demonstrated on CTA studies obtained prior to the study performed at the time of rupture. The mean growth for the aneurysms that ruptured was $4.25 \mathrm{~mm}$ (range $3-7 \mathrm{~mm}$, median $3.5 \mathrm{~mm}$ ). Four of six (66.7\%) ruptured aneurysms demonstrated multilobulated morphology compared with 16 of $174(9.1 \%)$ of unruptured 
Table 1 Summary of patient data and univariate analysis

\begin{tabular}{|c|c|c|c|c|c|c|}
\hline & Total population & Unruptured & Ruptured & $95 \% \mathrm{Cl}$ & OR & $\mathrm{p}$ Value \\
\hline Patients & $n=152$ & $n=146$ & $\mathrm{n}=6$ & & & \\
\hline Gender & & & & 0.051 to 1.347 & 0.261 & 0.109 \\
\hline Men & $36(23.7 \%)$ & $33(22.6 \%)$ & $3(50 \%)$ & & & \\
\hline Women & $116(76.3 \%)$ & $113(77.4 \%)$ & $3(50 \%)$ & & & \\
\hline Mean age (years) & 61.5 & 62.4 & 71.2 & 0.098 to 1.193 & 1.091 & 0.055 \\
\hline Current smoker & $32(17.8 \%)$ & $29(19.9 \%)$ & $3(50 \%)$ & 0.999 to 39.192 & 6.259 & 0.050 \\
\hline Hypertensive & $132(86.8 \%)$ & $128(87.7 \%)$ & $4(66.7 \%)$ & 0.462 to 15.384 & 2.667 & 0.273 \\
\hline Aneurysms & $n=180$ & $n=174$ & $\mathrm{n}=6$ & 0.009 to 3.240 & 0.175 & 0.242 \\
\hline Single & $129(84.9 \%)$ & $123(84.2 \%)$ & $6(100 \%)$ & & & \\
\hline Multiple & $23(15.1 \%)$ & $23(15.8 \%)$ & 0 & & & \\
\hline Aneurysm location & & & & 0.924 to 24.924 & 4.800 & 0.062 \\
\hline Anterior & $147(81.7 \%)$ & $145(83.3 \%)$ & $2(33.3 \%)$ & & & \\
\hline Posterior & $33(18.3 \%)$ & $29(16.7 \%)$ & $4(66.7 \%)$ & & & \\
\hline Mean aneurysm size (mm) & 5.6 & 5.4 & 13.7 & 1.048 to 1.251 & 1.145 & 0.003 \\
\hline Calcification & $9(5.0 \%)$ & $9(5.2 \%)$ & 0 & 0.034 to 16.548 & 0.746 & 0.853 \\
\hline Thrombosed & $8(4.4 \%)$ & $7(4.0 \%)$ & $1(16.7 \%)$ & 0.022 to 2.041 & 0.210 & 0.178 \\
\hline Multilobulated & $20(11.1 \%)$ & $16(9.1 \%)$ & $4(66.7 \%)$ & 3.352 to 116.362 & 19.75 & 0.001 \\
\hline Growth & $8(4.4 \%)$ & $4(2.3 \%)$ & $4(66.7 \%)$ & 11.905 to 606.905 & 85.00 & $<0.0001$ \\
\hline No of CTAs (mean) & 3.4 & 3.4 & 2.3 & & & \\
\hline Follow-up (years) & 3.4 & 3.4 & 2.5 & & & \\
\hline Ruptured aneurysms & $6(3.3 \%)$ & 0 & $6(100 \%)$ & & & \\
\hline
\end{tabular}

aneurysms $(p=0.001$, figure 2$)$. In three of the four multilobulated aneurysms that ruptured, multilobulated morphology was demonstrated on CTA studies obtained prior to the study performed at the time of rupture. A single ruptured aneurysm demonstrated neither interval growth nor multilobulated morphology on CTA. A history of smoking in patients with ruptured aneurysms approached statistical significance $(p=0.05)$.

The statistically significant risk factors from the univariate analysis were subsequently evaluated with a multivariate logistic regression analysis (table 3 ). The risk factors that remained significant following the multivariate analysis were aneurysm growth (OR 55.9, 95\% CI 4.47 to 700.08; $\mathrm{p}=0.002$ ) and aneurysm multilobulation (OR 17.4, 95\% CI 1.52 to 198.4 ; $\mathrm{p}=0.022)$.

Table 2 Patients with ruptured aneurysms

\begin{tabular}{|c|c|c|c|c|c|}
\hline Patient & Location & $\begin{array}{l}\text { Size } \\
(\mathrm{mm})\end{array}$ & Growth & Multilobulated & Reason followed \\
\hline 1 & PICA & 12 & Yes & No & $\begin{array}{l}\text { Too high-risk due } \\
\text { to medical } \\
\text { conditions }\end{array}$ \\
\hline 2 & $\begin{array}{l}\text { Basilar } \\
\text { tip }\end{array}$ & 14 & No & Yes & $\begin{array}{l}\text { Too high-risk due } \\
\text { to medical } \\
\text { conditions }\end{array}$ \\
\hline 3 & MCA & 15 & No & No & $\begin{array}{l}\text { Too high-risk due } \\
\text { to medical } \\
\text { conditions }\end{array}$ \\
\hline 4 & MCA & 9 & Yes & Yes & $\begin{array}{l}\text { Patient elected } \\
\text { observation }\end{array}$ \\
\hline 5 & PCom & 11 & Yes & Yes & $\begin{array}{l}\text { Patient refused } \\
\text { treatment }\end{array}$ \\
\hline 6 & PCom & 22 & Yes & Yes & $\begin{array}{l}\text { Patient refused } \\
\text { treatment }\end{array}$ \\
\hline
\end{tabular}

MCA, middle cerebral artery; PICA, posterior inferior cerebellar artery; PCom, posterior communicating artery.
An analysis of a subset of 30 cases was performed to assess interobserver variability and agreement; 53\% of size measurements showed no deviation from the original. The average measurement deviation was $0.6 \mathrm{~mm}$ (range $0.5-2 \mathrm{~mm}$ ). There was $100 \%$ agreement for categorizing growth versus no growth of aneurysms between follow-up CTA studies.

\section{DISCUSSION}

In our study of 180 UIA followed conservatively with serial CTA we found an overall rupture incidence of $3.3 \%$ and a rupture rate of $0.97 \%$ per year of CTA follow-up. The risk factors most predictive of aneurysm rupture during follow-up were interval aneurysm growth and multilobulated aneurysm morphology.

The overall aneurysm rupture rate and annual rupture rate during CTA follow-up in our population is comparable to those published in previous studies of UIA. ${ }^{8} 91314$ Our findings are also similar to the results of a recent meta-analysis of UIA which demonstrated that the annual incidence of aneurysm rupture was $1.2 \%$ for UIA observed during less than 5 years of follow-up. ${ }^{15}$ Our UIA rupture rate of $3.3 \%$ was more than double that observed by a recent CTA study focusing on UIA growth which reported an overall rupture rate of $1.6 \%{ }^{12}$ This could be related to fact that the aforementioned study included fusiform aneurysms in their analysis, which have a different pathogenesis from saccular aneurysms and may have a distinct natural history. ${ }^{16}$

We found that multilobulated aneurysm morphology was significantly associated with aneurysm rupture during CTA follow-up in both the univariate and multivariate logistic regression analyses. This finding is concordant with a prior study that analyzed the morphology of ruptured aneurysms using DSA at a single time point and demonstrated that multiple lobes are more commonly found in ruptured than in unruptured aneurysms $(26 \%$ vs $4 \%, \mathrm{p}<0.05) .{ }^{17}$ Our results are also similar to a recent study using a combination of DSA, CTA and MRA to follow up 

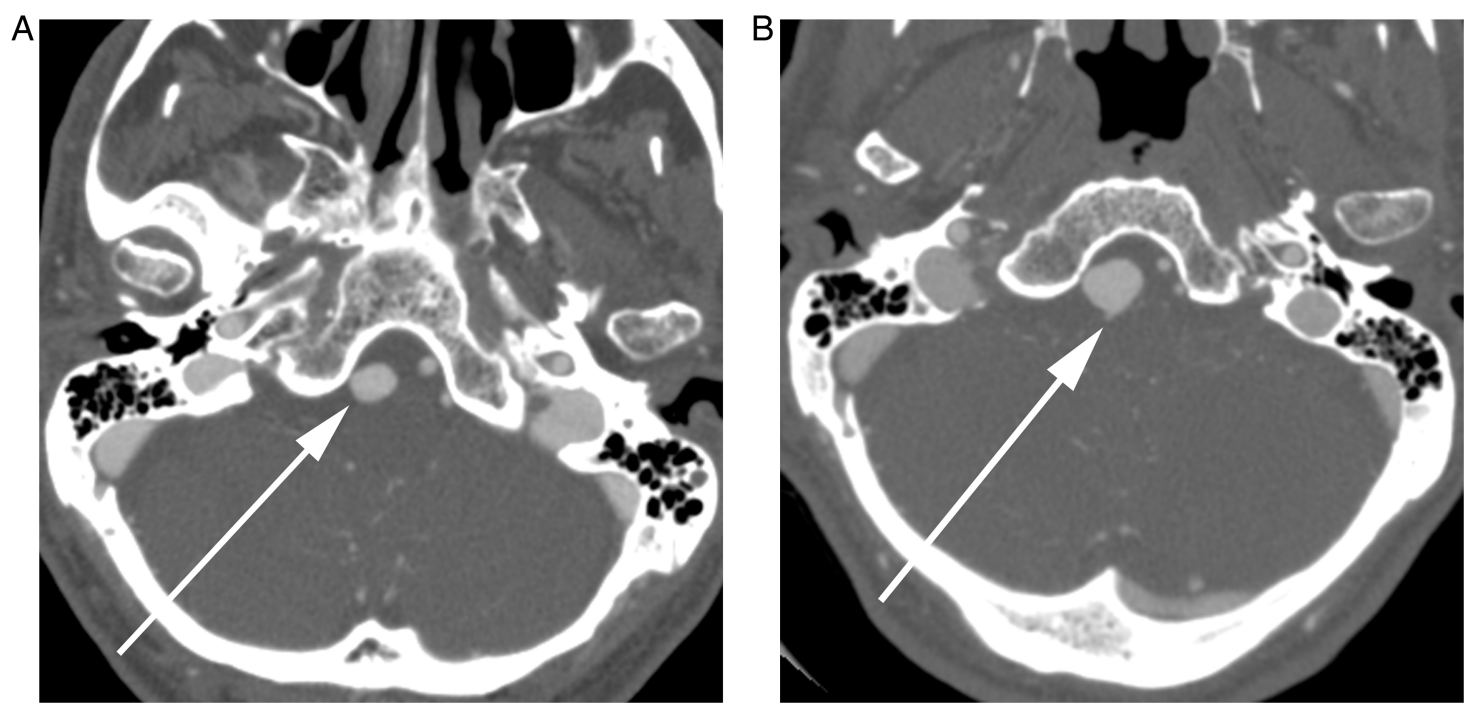

Figure 1 An elderly patient presented with dizziness and ataxia. (A) Axial CTA source image from initial study demonstrates an ovoid unilobular right posterior inferior cerebellar artery aneurysm (arrow) measuring $11 \mathrm{~mm}$ in maximum diameter. The patient was not offered treatment because the patient's age and aneurysm location were felt to be too high a risk. (B) Axial CTA source image from follow-up study obtained 2 years later at the time of rupture shows interval increase in size of the aneurysm (12 mm maximum diameter) with a new posterior outpouching (arrow).

UIA which also demonstrated a significant $(\mathrm{p}=0.02)$ association between the presence of an aneurysm daughter sac and rupture. $^{13}$ There is evidence that multilobulated aneurysm morphology increases local hemodynamic stress within aneurysms, which may explain the higher risk of aneurysm rupture. ${ }^{18}$

Interval aneurysm growth during serial CTA follow-up was the other factor significantly associated with rupture in both our univariate and multivariate analyses. We selected $2 \mathrm{~mm}$ as the criterion for growth in any dimension between examinations because we feel that there is a high degree of imprecision with smaller measurements. Smaller measurements are also difficult to translate into clinical practice since a change of $1 \mathrm{~mm}$ is considered by most radiologists to be within the domain of measurement error. Previous small studies using CTA and/or MRA to follow up UIA have focused on risk factors predicting interval aneurysm growth as a marker for aneurysm rupture. ${ }^{19-22}$ In contrast to our study, these studies did not specifically analyze the extent to which interval aneurysm growth predicted the risk of rupture. Our findings are similar to those of a recent North American CTA study of UIA which reported that aneurysm growth resulted in a 12-fold risk of subsequent rupture $(p=0.002){ }^{12}$ The increased risk of rupture in enlarging intracranial aneurysms may be related to increased hemodynamic stress with wall remodeling and weakness as the aneurysm grows in diameter.

Larger aneurysm size was significantly associated with rupture in our univariate analysis. All six ruptured aneurysms in our population were at least $9 \mathrm{~mm}$ in maximum diameter. This result is similar to the findings of multiple previous studies which demonstrated an increased risk of rupture of larger aneurysms, particularly in those measuring $>7 \mathrm{~mm}$ in diameter. ${ }^{5} 89$ $13-1523$
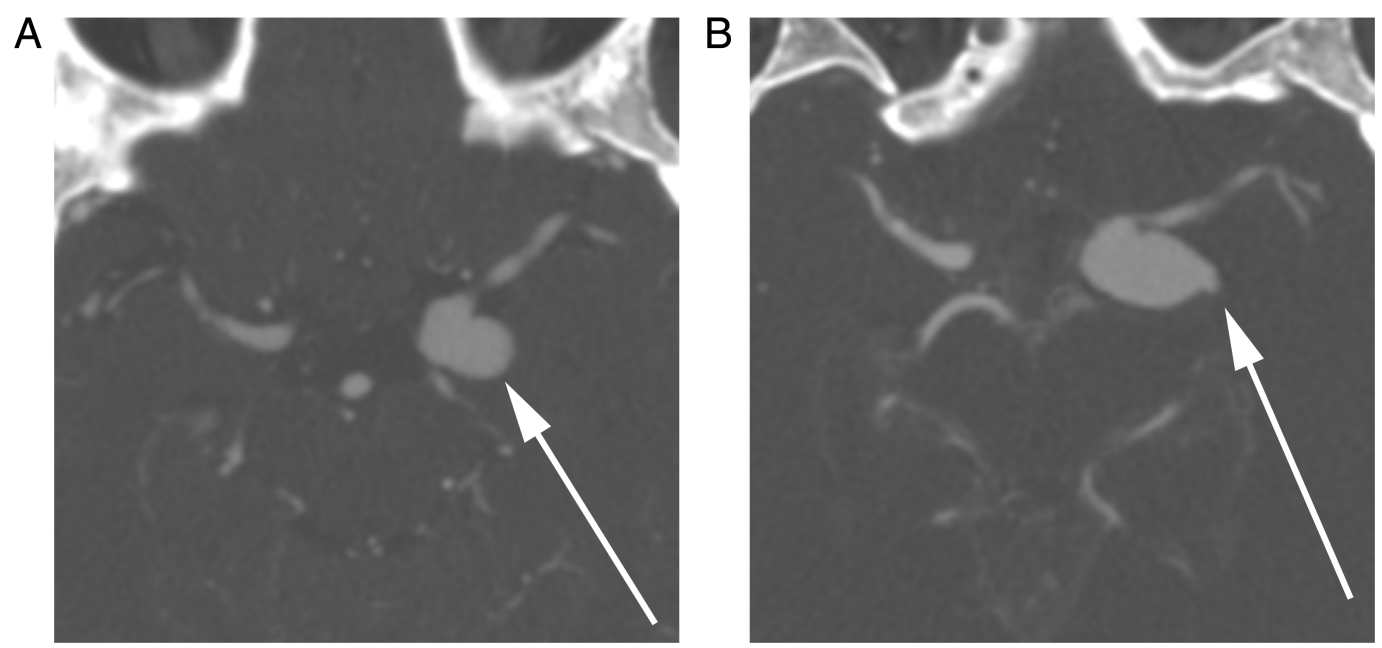

Figure 2 An elderly patient presented with dizziness. (A) Axial CTA source image from initial study demonstrates a bilobed left posterior communicating artery aneurysm (arrow) measuring $15 \mathrm{~mm}$ in maximum diameter. The patient refused treatment for the aneurym. (B) Axial CTA source image from follow-up study obtained 3 years later at the time of rupture shows interval increase in size of the aneurysm (22 mm maximum diameter) with a new posterolateral outpouching (arrow). 
Table 3 Multivariate analysis

\begin{tabular}{llll}
\hline Variable & $95 \%$ Cl & OR & p Value \\
\hline Aneurysm size & 0.947 to 1.257 & 1.091 & 0.230 \\
Growth & 4.469 to 700.084 & 55.93 & 0.002 \\
Multilobulated & 1.522 to 198.422 & 17.38 & 0.022 \\
\hline
\end{tabular}

There are reports of higher rates of aneurysmal subarachnoid hemorrhage in the Japanese population and genetic testing has identified two single nucleotide polymorphisms that predispose Japanese people to intracranial aneurysm formation. ${ }^{10} 11$ Although many recent UIA studies have been performed in Japanese patient populations, our results with an overall rupture rate of $3.3 \%$ are similar to or higher than the rupture rates observed in some Japanese studies. The reason for this is uncertain, but could be related to selection bias and more aggressive treatment practices at Japanese institutions.

Our study was limited by its retrospective methodology. As such, there is definite selection bias based on practitioner/patient choice to undergo conservative therapy. Additionally, the number of patients is smaller than in some of the natural history studies referenced above. It is possible that, despite our diligent review of the medical and radiographic records, patients were lost to follow-up.

While the number of ruptured lesions $(n=6)$ is not large, clinically meaningful parameters were detected by the univariate analysis. Moreover, multilobulation and growth remained significant in the multivariate logistic model. The only parameter that lost significance in the multivariate model was the size of the aneurysm. Although it is true that the power of this test was not large, the multivariate logistic model by itself exploited potential redundancy between the aneurysm size and other parameters. We believe that the small number of ruptured lesions was not a major weakness of the performed data analysis.

Another potential limitation is that we used the individual aneurysm as the unit of observation as opposed to the individual patient, which could have more heavily weighted patients with multiple UIA. However, this was unlikely to be an important factor since multiplicity of intracranial aneurysms was not demonstrated to be a significant independent predictor of intracranial aneurysm rupture. Furthermore, there is non-uniformity among the diagnostic neuroradiology staff at our tertiary care institution which reflects the national heterogeneity on what size criteria they would require to mention an aneurysm in the formal report. Additionally, patients with 'small aneurysms' might not get followed in this real-world retrospective series and this would have the potential to affect the statistical analysis.

Referral bias is another limitation. This study was conducted at a major regional cerebrovascular referral center for the management of UIA, which may have increased the proportion of complex aneurysms in our patient population. Furthermore, it is possible that more complex UIA were treated and therefore removed from our population of conservatively followed aneurysms. All of the previous studies of UIA suffer from a similar selection bias. It would be unethical to prospectively follow up large sized, growing and multilobulated aneurysms that would be otherwise treated. The relatively small number of observed ruptured intracranial aneurysms may also have limited our ability to detect additional significant independent risk factors for aneurysm rupture. Multiple previous studies have demonstrated a significantly increased risk of rupture in current smokers, patients with a history of hypertension, multiple aneurysms and posterior circulation aneurysms. ${ }^{6-8} \quad \begin{array}{llll}14 & 15 & 23\end{array}$ Lastly, the relatively long timeframe of our study over a 10 -year period introduced heterogeneity with respect to our CTA imaging hardware, software and protocols, which have evolved over time. However, all CTA studies were performed on either 16- or 64-detector row CT scanners produced by the same manufacturer.

The non-invasive nature of CT angiography coupled with its relatively high spatial resolution, speed and availability make CTA an excellent modality for the serial follow-up of known UIA. Based on our findings, UIA with a multilobulated morphology and/or interval growth between follow-up CTA studies are at increased risk of rupture. Such aneurysms should be considered for definitive treatment rather than continued observation with imaging, given the potentially devastating consequences of rupture. Further studies of conservative CTA follow-up of UIA with longer follow-up periods and larger numbers of ruptured aneurysms are necessary. Finally, we acknowledge that there are different ways to follow UIA. In this era of heightened radiation awareness one could certainly use MRA. We think this is a reasonable approach but prefer CTA because of its rapidity, spatial resolution and uniformity over time.

\section{CONCLUSION}

CTA is a widely available, safe and low-cost imaging technique that is increasingly being performed for the surveillance of UIA. This study demonstrates that multilobulated morphology and interval growth of UIA are characteristics predictive of a higher risk of subsequent rupture during conservative CTA follow-up. The presence of these features could help guide clinical decisions as to whether these aneurysms are followed conservatively or treated.

Intracranial aneurysms are characteristics predictive of a higher risk of subsequent rupture during conservative CTA follow-up. The presence of these features could help guide clinical decisions as to whether these aneurysms are followed conservatively or treated.

Acknowledgements The authors thank Nina N Waldron, Ultrasound Technician Assistant, for her assistance with collecting patient clinical data, Hang Lee PhD for his assistance with the statistical evaluation and Jason M Johnson MD for his help with preparing the figures.

Contributors All authors meet ICMJE guidelines for authorship. WAM: developed and implemented the research plan, collected and analyzed the data, wrote the rough draft and edited the manuscript. JMR: conceptualized the project and developed the research plan, assisted in data analysis and edited the drafts and final manuscript. JAH: developed the research plan and edited the drafts and final manuscript. DJS: developed the research plan, collected and organized the aneurysm database and edited the drafts and final manuscript. RGG: conceptualized the project and developed the research plan and edited the drafts and final manuscript. $\mathrm{JJH}$ : helped to develop the research plan, organized the aneurysm database and edited the drafts and final manuscript. PWS: leader of the project; conceptualized the project and developed the research plan, assisted with data analysis and edited the drafts and final manuscript.

Funding This work was conducted with support from Harvard Catalyst grant number 8UL1TR000170-05.

Competing interests None.

Ethics approval This study was compliant with the Health Insurance Portability and Accountability Act (HIPAA) and was approved by the Institutional Review Board of Massachusetts General Hospital.

Provenance and peer review Not commissioned; externally peer reviewed. 


\section{REFERENCES}

1 Rinkel GJ, Djibuti M, Algra A, et al. Prevalance and risk of rupture of intracranial aneurysms: a systematic review. Stroke 1998;29:251-6.

2 Winn HR, Jane JA Sr, Taylor J, et al. Prevalence of asymptomatic incidental aneurysms: review of 4568 arteriograms. J Neurosurg 2002;96:43-9.

3 Vlak MH, Algra A, Bradenburg R, et al. Prevalance of unruptured intracranial aneurysms, with emphasis on sex, age, comorbidity, country and time period: a systematic review and meta-analysis. Lancet Neurol 2011;10:626-36.

4 Loumiotis I, Brown RD Jr, Vine R, et al. Small $(<10-\mathrm{mm})$ incidentally found intracranial aneurysms, Part 2: treatment recommendations, natural history, complications and short-term outcome in 212 consecutive patients. Neurosurg Focus 2011;31:E4.

5 Wiebers DO, Whisnant JP, Huston J III, et al Unruptured intracranial aneurysms: natural history, clinical outcome, and risks of surgical and endovascular treatment. Lancet 2003;362:103-10.

6 Vlak MH, Rinkel GJ, Greebe P, et al. Independent risk factors for intracranial aneuryms and their joint effect. Stroke 2013;44:984-7.

7 Nahed BV, DiLuna ML, Morgan T, et al. Hypertension, age, and location predict rupture of small intracranial aneurysms. Neurosurgery 2005;57:676-83.

8 Juvela S, Porras M, Poussa K. Natural history of unruptured intracranial aneurysms: probability of and risk factors for aneurysm rupture. J Neurosurg 2008;108:1052-60.

9 Ishibashi T, Murayama Y, Urashima M, et al. Unruptured intracranial aneurysms: incidence of rupture and risk factors. Stroke 2009;40:313-16.

10 Low SK, Takahashi A, Cha PC, et al. Genome-wide association study for intracranial aneurysm in the Japanese population identifies three candidate susceptibility loci and a functional variant at EDNRA. Hum Mol Genet 2012;21:2102-10.

11 Morita A, Fujiwara S, Hashi K, et al. Risk of rupture associated with intact cerebral aneurysms in the Japanese population: a systematic review of the literature from Japan. J Neurosurg 2005;102:601-6.

12 Villablanca JP, Duckwiler G, Jahan R, et al. Natural history of asymptomatic unruptured cerebral aneurysms evaluated at CT angiography: growth and rupture incidence and correlation with epidemiologic risk factors. Radiology 2013;269:258-65.

13 Morita A, Kirino T, Hashi K, et al. The natural course of unruptured cerebral aneurysms in a Japanese cohort. N Engl J Med 2012;366:2474-82.

14 Sonobe M, Yamazaki T, Yonekura M, et al. Small Unruptured Intracranial Aneurysm Verification Study: SUAVe study, Japan. Stroke 2010;41:1969-77.

15 Wermer MJ, van der Schaaf IC, Algra A. Risk of rupture of unruptured intracranial aneurysms in relation to patient and aneurysm characteristics: an updated meta-analysis. Stroke 2007;38:1404-10.

16 Park SH, Yim MB, Lee CY, et al. Intracranial fusiform aneurysms: its pathogenesis, clinical characteristics and managements. J Korean Neurosurg Soc 2008;44: 116-23.

17 Beck J, Rohde S, el Beltagy M, et al. Difference in configuration of ruptured and unruptured intracranial aneurysms determined by biplanar digital subtraction angiography. Acta Neurochir 2003;145:861-5.

18 Meng H, Feng Y, Woodward SH, et al. Mathematical model of the rupture mechanism of intracranial saccular aneurysms through daughter aneurysm formation and growth. Neurol Res 2005;27:459-65.

19 Burns JD, Huston J III, Layton KF, et al. Intracranial aneurysm enlargement on serial magnetic resonance angiography: frequency and risk factors. Stroke 2009;40:406-11.

20 Phan TG, Huston J III, Brown RD Jr, et al Intracranial saccular aneurysm enlargement determined using serial magnetic resonance angiography. J Neurosurg 2002;97:1023-8

21 Miyazawa N, Akiyama I, Yamagata Z. Risk factors for growth of unruptured intracranial aneurysms: follow-up study by serial 0.5-T magnetic resonance angiography. Neurosurgery 2006;58:1047-53.

22 Matsubara S, Hadeishi $H$, Suzuki $A$, et al. Incidence and risk factors for the growth of unruptured cerebral aneurysms: observation using serial computerized tomography angiography. J Neurosurg 2004;101:908-14.

23 Juvela S, Poussa K, Porras M. Factors affecting formation and growth of intracranial aneurysms: a long-term follow-up study. Stroke 2001;32:485-91. 